\title{
Geoprocessamento da tuberculose no município de Santos - SP entre 2006 e 2016
}

\author{
Tuberculosis geoprocessing in the city of Santos - SP between 2006 and 2016 \\ Geoprocessamiento de tuberculosis em la ciudad de Santos - SP entre 2006 y 2016
}

\section{Resumo}

Realizar um Geoprocessamento da Tuberculose no município de Santos no período compreendido entre 2006 e 2016 a fim de se identificar as regiões com maior número de casos do município. O presente estudo caracteriza-se como ecológico e de tendencia temporal, uma vez que avalia de forma retrospectiva todos os casos de Tuberculose no município de Santos entre 2006 e 2016. Os dados obtidos foram cedidos pelo Centro de Vigilância Epidemiológica do estado de São Paulo, sendo fornecido pelo CVE-SP. A base de dados foi composta de 4533 casos para o município de Santos. A maior concentração de casos observada ocorreu na zona Noroeste de Santos, composta pelos bairros Rádio Clube, Chico de Paula e Bom retiro, a região portuária composta pelo bairro do Paquetá e Vila Nova. Uma terceira área de concentração de casos está localizada na região composta pelo morro Monte Serrat, Vila Progresso e Saboó. bairros do Paquetá, Vila Matias e Vila Nova (81 casos) e a região composta pelos bairros Alemoa, Radio Clube, Bom Retiro e São Jorge (103 casos). Essas duas principais regiões totalizaram juntas 184 casos, representando aproximadamente $62 \%$ de todos os casos observados no município. Foram identificadas três regiões principais que contribuíram para a maioria dos casos no município, sendo elas, a zona noroeste, a região portuária do Paquetá e a região de morros da nova Cintra e Monte Serrat. Políticas públicas voltadas para essas três regiões poderiam evitar a persistência da disseminação de casos entre elas.

Palavras-chave: Tuberculose; Geoprocessamento; Epidemiologia.

\begin{abstract}
To perform a Tuberculosis Geoprocessing in the city of Santos in the period between 2006 and 2016 in order to identify the regions with the highest number of cases in the city. The present study is characterized as ecological and temporal trend, as it retrospectively evaluated all cases of Tuberculosis in the city of Santos between 2006 and 2016. The requested data were provided by the Epidemiological Surveillance Center of the state of São Paulo, being provided by CVE-SP. The database consisted of 4533 cases for the city of Santos Results: The highest concentration of cases occurred in the Northwest of Santos, comprising the districts Rádio Clube, Chico de Paula and Bom Retreat, a port region in the neighborhood of Paquetá and Vila Nova. A third area of concentration of cases is located in the region comprising Monte Serrat, Vila Progresso and Saboó. neighborhoods of Paquetá, Vila Matias and Vila Nova (81 cases) and the region made up of the neighborhoods Alemoa, Radio Clube, Bom Retiro and São Jorge (103 cases). These two main regions totaled 184 cases, representing approximately $62 \%$ of all cases observed in the city. Three main regions were identified that contributed to most of the cases in the city, namely, the northwest zone, the port
\end{abstract}


region of Paquetá and the region of hills of Nova Cintra and Monte Serrat. Public policies aimed at these three regions can prevent the persistence of the spread of cases between them.

Keywords: Tuberculosis; Geoprocessing; Epidemiology.

\begin{abstract}
Resumen
Realizar un Geoprocesamiento de Tuberculosis en la ciudad de Santos en el período 2006-2016 con el fin de identificar las regiones con mayor número de casos en la ciudad. El presente estudio se caracteriza como tendencia ecológica y temporal, ya que evaluó retrospectivamente todos los casos de Tuberculosis en la ciudad de Santos entre 2006 y 2016. Los datos solicitados fueron proporcionados por el Centro de Vigilancia Epidemiológica del estado de São Paulo, siendo proporcionados por CVE-SP. La base de datos constó de 4533 casos para la ciudad de Santos. La mayor concentración de casos ocurrió en el Noroeste de Santos, que comprende los distritos Rádio Clube, Chico de Paula y Bom Retreat, una región portuaria en el barrio de Paquetá y Vila Nova. Una tercera área de concentración de casos se localiza en la comarca que comprende Monte Serrat, Vila Progresso y Saboó. barrios de Paquetá, Vila Matias y Vila Nova (81 casos) y la región formada por los barrios Alemoa, Radio Clube, Bom Retiro y São Jorge (103 casos). Estas dos regiones principales totalizaron 184 casos, lo que representa aproximadamente el $62 \%$ de todos los casos observados en la ciudad. Se identificaron tres regiones principales que contribuyeron a la mayoría de los casos en la ciudad, a saber, la zona noroeste, la región portuaria de Paquetá y la región de cerros de Nova Cintra y Monte Serrat. Las políticas públicas dirigidas a estas tres regiones pueden evitar que persista la propagación de casos entre ellas.
\end{abstract}

Palabras clave: Tuberculosis; Geoprocessamiento; Epidemiologia.

\title{
1. Introdução
}

A Tuberculose (TB) configura ainda um grave problema de saúde pública a nível nacional e internacional. A doença está intimamente ligada a questões econômicas e distribuição socioespacial (Leal, 2018). Segundo o Global Tuberculosis Report 2017, da Organização Mundial da Saúde (OMS), a tuberculose é a nona causa de morte no mundo. No ano de 2016, 10,4 milhões de pessoas manifestaram a doença e 1,7 milhão vieram a óbito devido a esta. Estima-se que $25 \%$ da população mundial apresente a forma latente da TB e que 5-15\% desta irá desenvolver manifestações clínicas ao longo da vida (WHO, 2017). O Brasil ocupa, atualmente a 16 posição em carga de Tuberculose, representando 0,9\% dos casos estimados no mundo e $33 \%$ dos casos estimados para as américas (Rocha, 2000), (Ximenes et al, 1999).

No Brasil, pouco se sabe sobre a distribuição espacial das doenças endêmicas em áreas urbanas. A distribuição espacial das doenças endêmicas, como a $\mathrm{TB}$, constitui objeto de estudo da epidemiologia geográfica. A aplicação do geoprocessamento a saúde torna-se uma poderosa ferramenta para conexão entre saúde e ambiente, a produção de mapas permite visualizar situações de risco a saúde (Barcellos \& Santos 1997). Nesse sentido, o espaço surge como categoria de estudo, na perspectiva de melhor compreender o processo saúde-doença nas coletividades através da distribuição e caracterização epidemiológica da ocorrência de endemias em áreas urbanas (Leal, 2018).

Estudos mostram que a saúde da população apresenta um gradiente social, desfavorecendo os grupos socialmente menos privilegiados. Em relação a Tuberculose (TB), várias pesquisas vêm demonstrando sua relação com as condições de vida, destacando o papel dos indicadores de desigualdade social com a ocorrência de doença (Câmara et al., 2001), (Souza et al, 2007), cujas populações socialmente desfavorecidas são atingidas de forma intensa e desproporcional pela TB.

O termo geoprocessamento é definido como um conjunto de coleta de dados (Câmara et al., 2001) que, em conjunto, produz informações demográficas e contribuem para o reconhecimento das determinadas condições de risco de um território (Souza et al, 2007). O geoprocessamento se tornou uma ferramenta eficaz para elaboração de mapas, permitindo a demonstração geoespacial dos fenômenos epidemiológicos que ocorrem naquela região (Hino et al, 2008).

Apesar do declínio observado nos últimos anos (2000-2004), os níveis de morbidade e mortalidade por tuberculose pulmonar verificados no município de Santos apontam a gravidade da situação. As taxas medias de incidência e de mortalidade superam amplamente as verificadas, em média, no estado de São Paulo e no país (Vieira et al, 2009). Sendo assim, o estudo da distribuição geoespacial dos casos de tuberculose permitiria a identificação das macrorregiões de maior prevalência da doença, 
possibilitando maiores investimentos locais para controle de transmissão e acompanhamento de pacientes contaminados pelo bacilo.

As taxas de incidência média segundo a região de residência mostraram uma distribuição desigual, variando de 72,8 a 272,9/100.000 habitantes na Orla Marítima e no Centro/Porto (Vieira et al, 2009), respectivamente, mostrando a necessidade de uma análise geoespacial da distribuição dos casos dentro de um município.

\section{Metodologia}

O município de Santos situa-se a $23^{\circ} 56^{\prime} 26^{\prime \prime}$ de latitude Sul e 46 $19^{\prime}$ '47' de longitude oeste nas regiões Sudeste do estado de São Paulo e Sudeste brasileira e, segundo o Instituto de Brasileiro de Geografia e Estatística (IBGE), em 2020 contava com 433.656 habitantes. A área total é de 231,6 km², implicando em uma densidade demográfica de 1545,1 hab $/ \mathrm{km}^{2}$.

O presente estudo caracteriza-se como ecológico e de tendencia temporal, uma vez que avalia de forma retrospectiva todos os casos de Tuberculose no município de Santos entre 2006 e 2016 através do emprego de variáveis qualitativas (Pereira, A. S et al, 2018). Os dados obtidos foram cedidos pelo Centro de Vigilância Epidemiológica do estado de São Paulo, sendo fornecido pelo CVE-SP uma planilha de Excel contendo todos os casos de Tuberculose diagnosticadas em Santos entre 2006 e 2016.

A base de dados foi composta de 4533 casos para o município de Santos, sendo que cada paciente possui seu número de identificação SINAN. Não foram aceitos casos de tuberculose diagnosticados em outros municípios, fora da década estipulada na pesquisa e moradores de demais localidades. A população de rua foi excluída deste estudo por não apresentar residência fixa, assim como os campos sem informação de endereço, totalizando 233 pacientes excluídos do trabalho.

Os endereços fornecidos na planilha para cada caso de Tuberculose foram transferidos para o Google Maps a fim de se obterem dados de latitude e longitude. Os dados que não puderam ser exportados foram codificados manualmente conforme o logradouro contido na planilha. Caso o endereço correto não fosse obtido, era atribuído um endereço próximo ao selecionado.

Em seguida, os dados espaciais foram tratados na plataforma Excel e transformados em Shapefile para exportação para a plataforma de georreferenciamento QGIS 3.4. A plataforma de geoprocessamento QGIS 3.4 foi escolhida para análise dos resultados obtidos, fornecendo mapas contendo todos os casos de Tuberculose e as variáveis selecionadas. A base cartográfica utilizada para divisão do município em bairros foi obtida através da plataforma SigSantos e através da plataforma online do IBGE foram obtidos os dados de aglomerados subnormais do município de Santos.

As variáveis adotadas para compor o estudo foram: Sexo (masculino e feminino), Desfecho (cura, óbito, abandono de tratamento), Drogadição, HIV (positivo, negativo, sem informação). Cada uma delas foi utilizada para confecção dos mapas

Após georreferenciamento dos casos, foram obtidos padrões de densidades espaciais por meio da análise estatística espacial, segundo a técnica de alisamento Kernel do software QGIS 3.4. Esse estimador considerou os casos de Tuberculose vizinhos ocorridos em determinado raio e tem como parâmetros básico o raio de influência, quanto maior a concentração de casos, mais escuro é o tom apresentado na área, sendo o método escolhido como preferencial para análise dos resultados.

Este estudo foi submetido e aprovado pelo comitê de ética em pesquisa do Centro Universitário Lusíada (Fundação

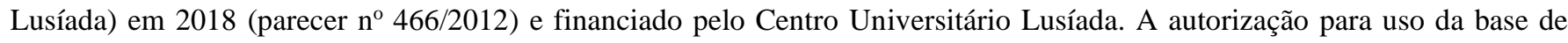
dados junto ao CVE-SP foi obtida mediante carta de Anuência, sendo resguardado sigilo dos dados de identificação dos pacientes. 


\section{Resultados e Discussão}

A Figura 1 mostra a distribuição dos casos de tuberculose após georreferenciamento para toda a década estudada. Podemos identificar uma maior concentração de casos em três regiões especificas do município de Santos. A maior concentração de casos observada ocorreu na zona Noroeste de Santos, composta pelos bairros Radio Clube, Chico de Paula e Bom retiro. Essa região é composta por uma diversidade de comunidades carentes e uma extensa área de palafitas em toda faixa litorânea da região. A região portuária composta pelo bairro do Paquetá e Vila Nova é marcada pela presença histórica de uma concentração populacional em habitações chamadas de cortiços, por isso é uma região com elevados números de casos de tuberculose em Santos. Uma terceira área de concentração de casos está localizada na região composta pelo morro Monte Serrat, Vila Progresso e Saboó.

Ainda que o mapa de pontos seja importante para a visualização dos casos, optou-se também por apresentar o mapa Kernel, que permite uma análise dos casos baseada em áreas de influência, facilitando a interpretação. A Figura 2 mostra a distribuição dos casos de tuberculose no município de Santos através de mapa de calor pelo método Kernel, com uma maior presença de cores mais quentes nas regiões Oeste e Nordeste do município, evidenciando a maior concentração de casos.

Figura 1: Casos de tuberculose notificados para o município de Santos entre 2006 e 2016 após georreferenciamento.

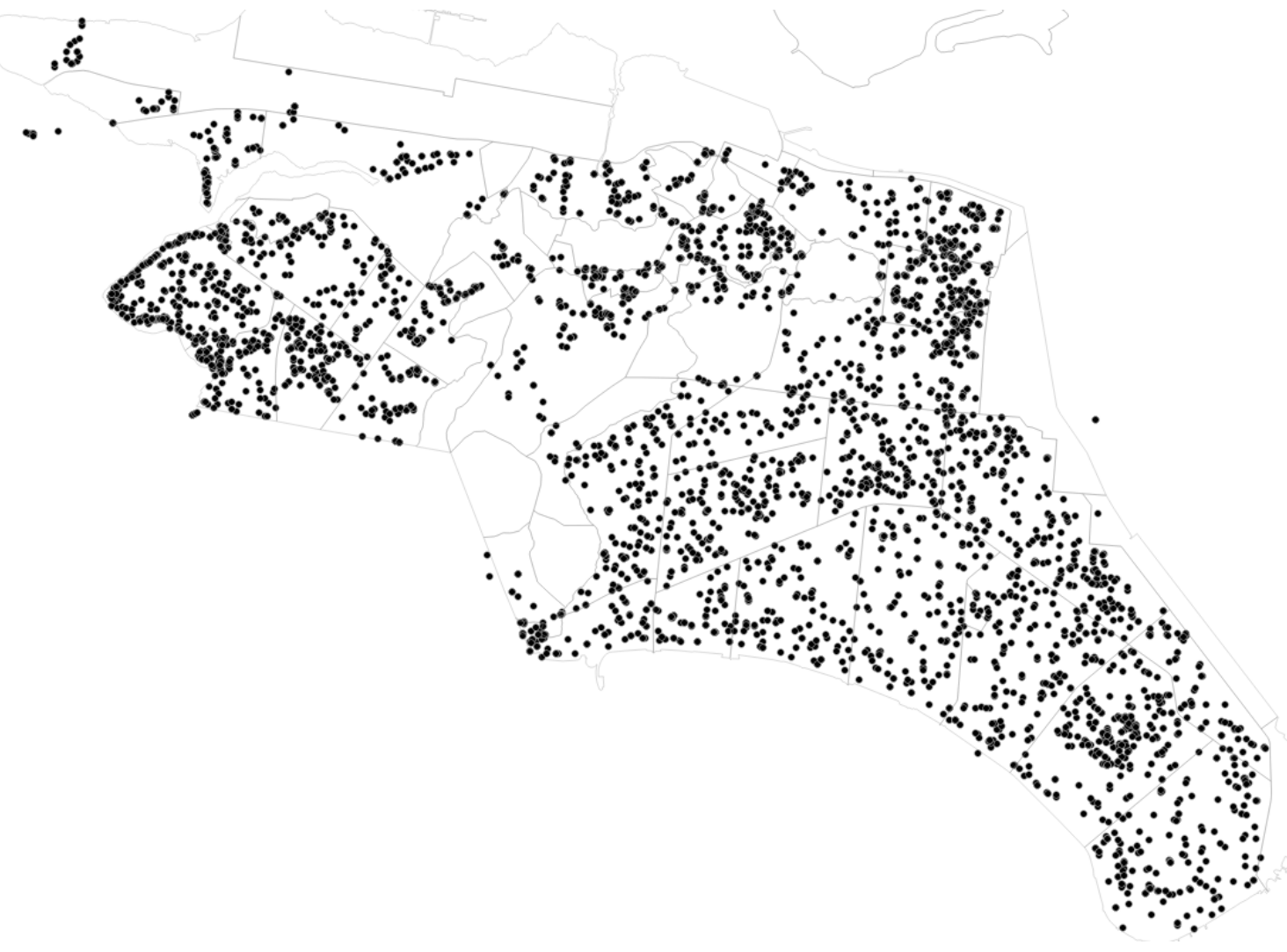

Fonte: Autores. 
Research, Society and Development, v. 11, n. 1, e44311125287, 2022

(CC BY 4.0) | ISSN 2525-3409 | DOI: http://dx.doi.org/10.33448/rsd-v11i1.25287

Figura 2: distribuição dos casos de tuberculose no município de Santos através de mapa de calor pelo método Kernel

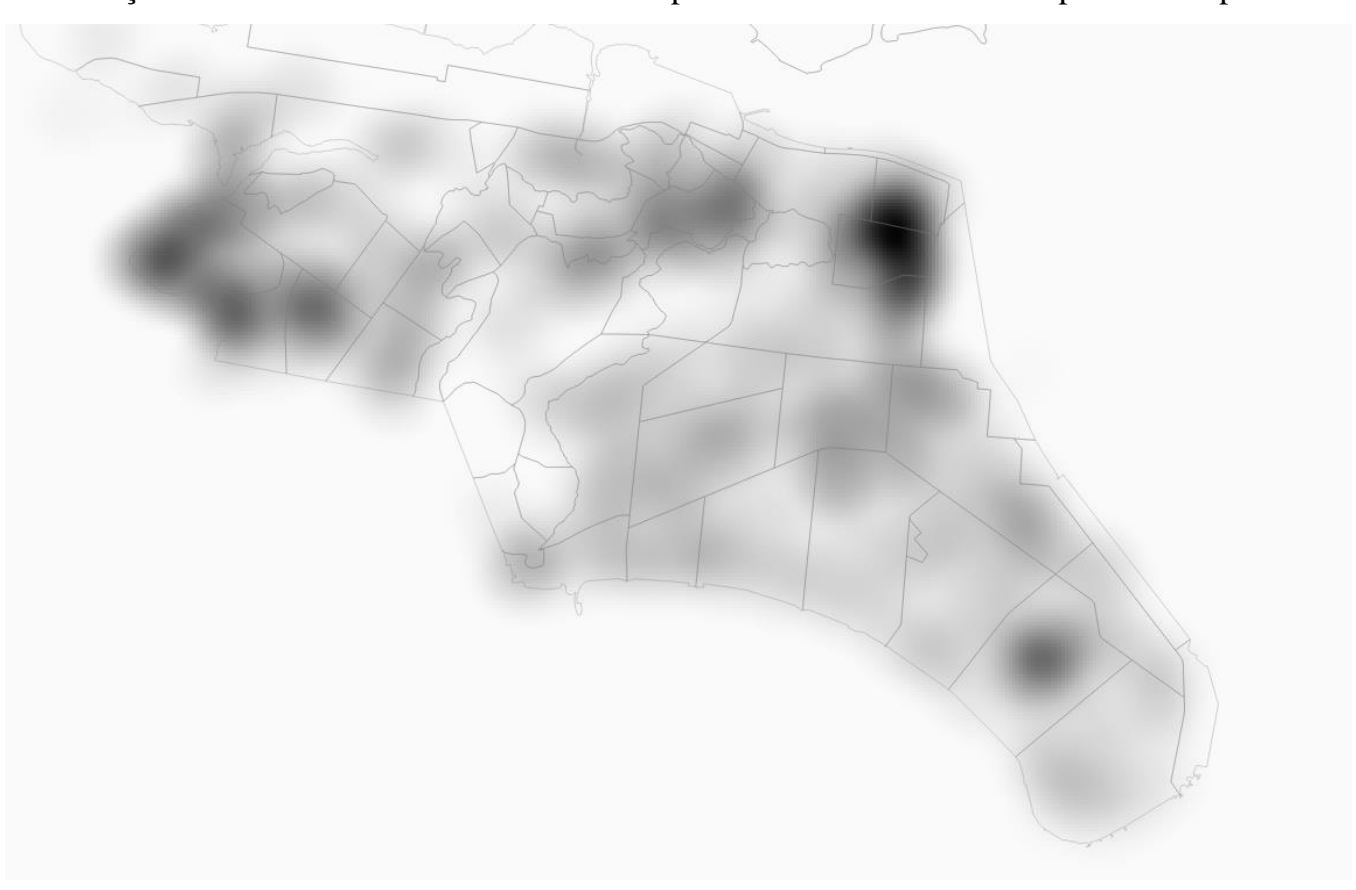

Fonte: Autores.

Ao comparar o número de casos ao longo dos anos através de mapas de calor pelo método Kernel, observamos na Figura 3 que no início da década os casos se apresentaram distribuídos de forma mais homogênea pelo município de Santos. A partir de 2012, duas regiões passaram a apresentar uma maior concentração de casos, sendo elas a zona Noroeste do município e a zona portuária do Paquetá. 
Figura 3: Evolução do número de casos no município de Santos ao longo da década avaliada (2006-2016) representada em 10 mapas através do método de Kernel.
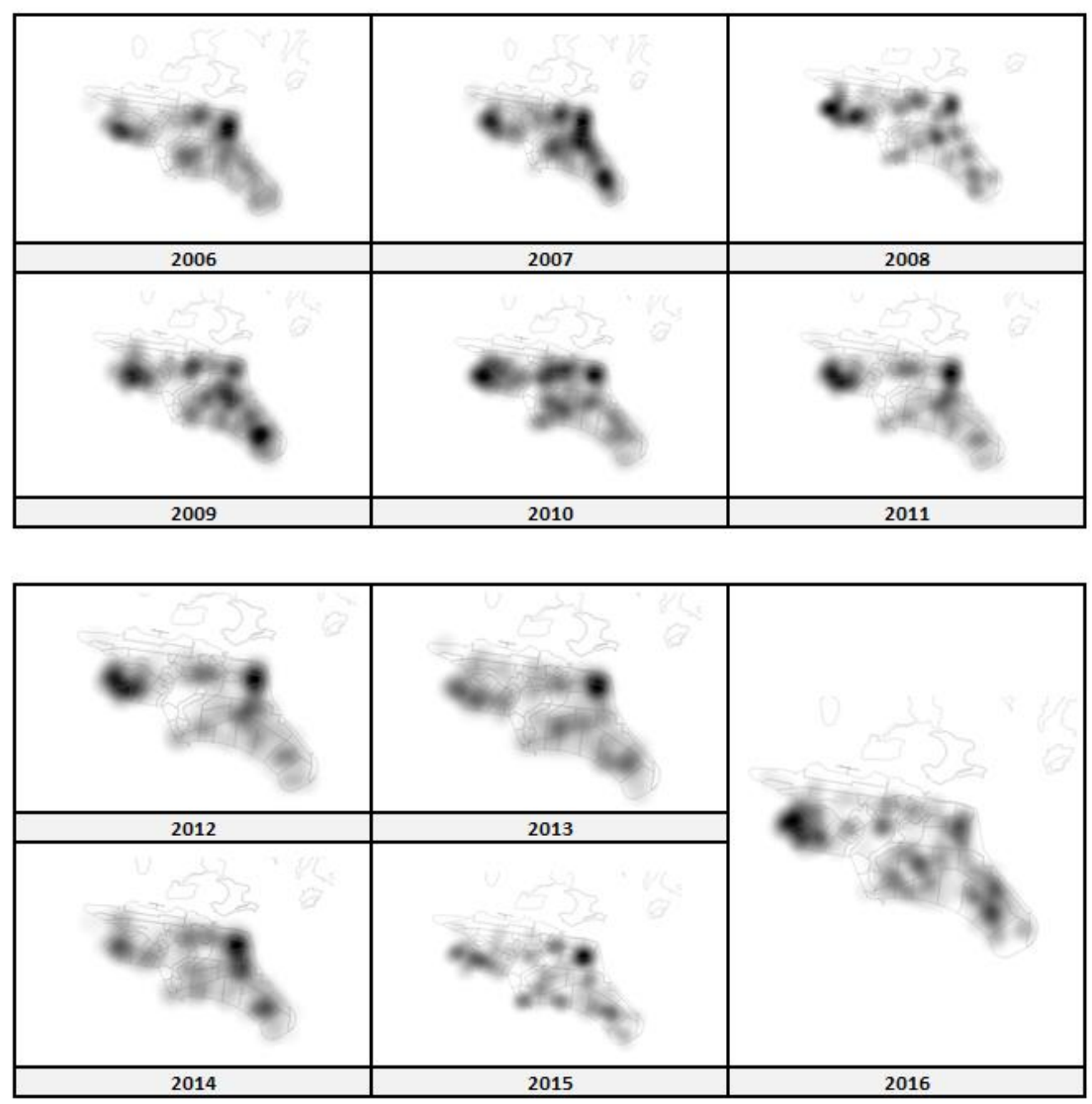

Fonte: Autores.

$\mathrm{Na}$ tentativa de comparar as regiões que compõem o município de Santos, a (Figura 4) busca mostrar através do método de "pontos de aglomeração" da plataforma Qgis quais as regiões com maior aglomeração de casos para toda a década avaliada. Notamos que as regiões com maior aglomeração ocorreram no bairro do Paquetá com 653 casos (região portuária), na região de morro do Monte Serrat (638 casos) e nos bairros Chico de Paula, Radio Clube e Bom Retiro (1039 casos). As menores taxas de aglomeração observadas ocorreram nos bairros do Gonzaga (199 casos) e na região da ponta da Praia (176 casos), áreas compostas por uma população com maior poder aquisitivo e menor concentração populacional por metro quadrado. Portanto, observamos que existe uma tendencia de sobreposição entre as aglomerações de casos observados com as regiões ocupadas por "aglomerados subnormais", principalmente na região Oeste do município e na região do morro de Monte Serrat.

Ao somar o número de casos dessas três principais regiões de aglomeração, notamos que somadas elas representam aproximadamente $51 \%$ dos casos, ou seja, metade dos casos do município de Santos se encontram nas regiões de maior aglomeração populacional e maiores níveis de pobreza. 
Figura 4: "Método de pontos de aglomeração" para mostrar as áreas de Santos com maior aglomeração de número de casos para toda a década avaliada e as regiões denominadas "Aglomerados subnormais" destacando áreas de moradia irregular e áreas de pobreza.

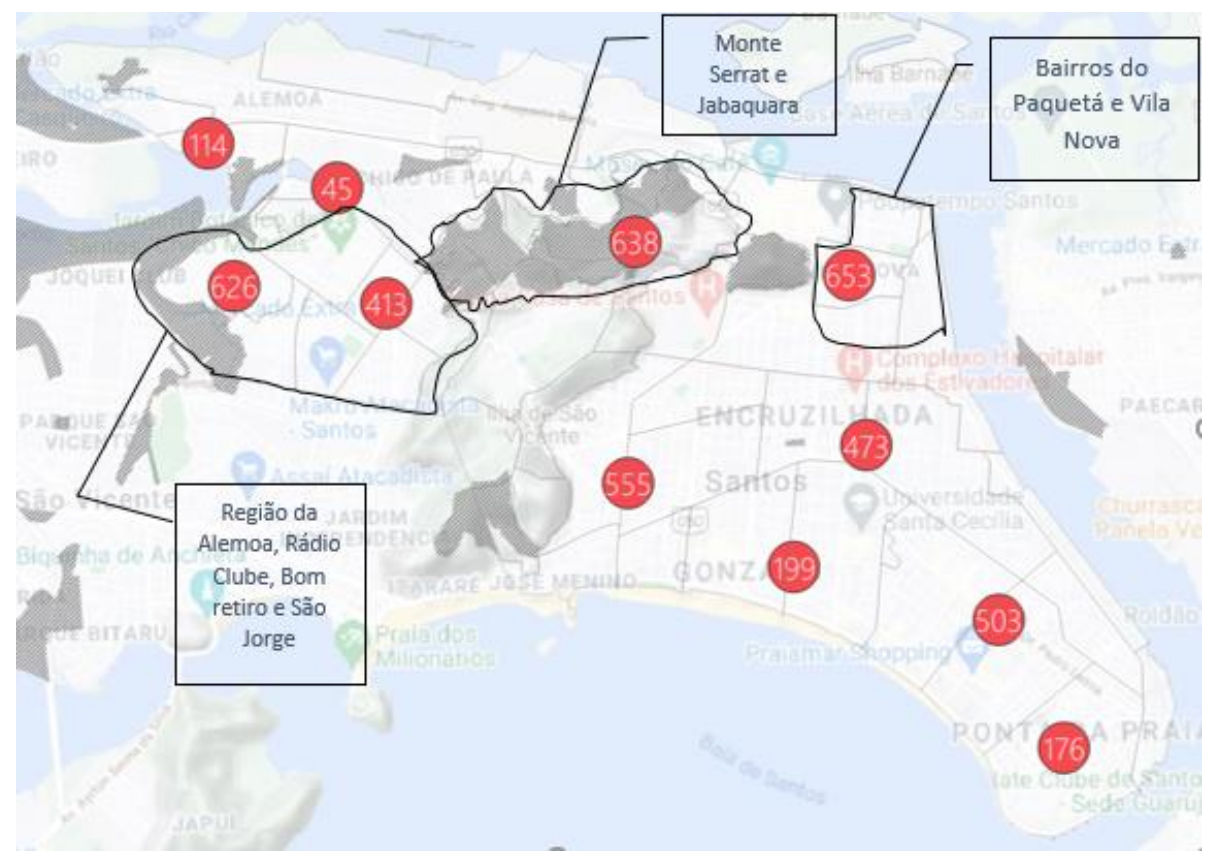

Fonte: Autores.

A variável DROGADIÇÃO presente na base de dados permitiu a identificação dos bairros com maior taxa da associação entre a tuberculose e o uso de drogas. Com isso, a Figura 5 mostrou um total de 295 casos dessa associação, as duas regiões com maiores taxas de aglomeração observada pelo método de pontos de aglomeração foram a dos bairros do Paquetá, Vila Matias e Vila Nova (81 casos) e a região composta pelos bairros Alemoa, Radio Clube, Bom Retiro e São Jorge (103 casos). Essas duas principais regiões totalizaram juntas 184 casos, representando aproximadamente $62 \%$ de todos os casos observados no município. 
Figura 5: Associação da variável DROGADIÇÃO com os casos de tuberculose, demostrado pelo método "pontos de aglomeração", mostrando as duas regiões com maiores taxas de aglomeração.

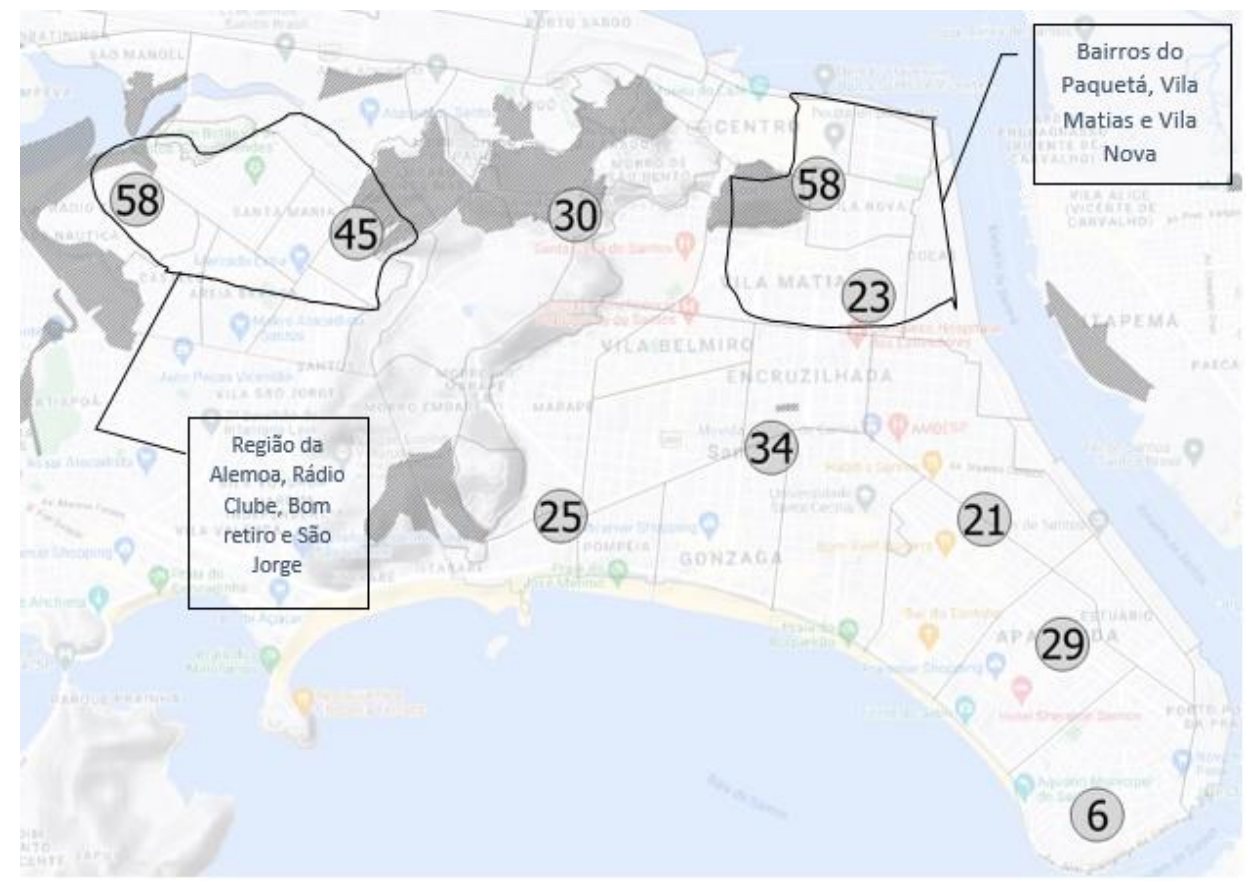

Fonte: Autores.

Neste estudo, a visualização de mapas de pontos, mapa de calor pelo método de Kernel ou através dos mapas de aglomeração, permitiram uma melhor exploração visual sobre como os eventos estao interligados ao espaço, bem como a evolução dos casos ao longo da década.

A TB está intimamente ligada a situação socioeconômica de uma região, sendo então uma enfermidade em que múltiplos fatores causais estao relacionados, ao lado do próprio bacilo, para determinação da doença (Dos santos \& Hino 2004). A criação de bolsões de pobreza nas áreas urbanas propicia o surgimento de moradias improprias e aglomerados populacionais, favorecendo a disseminação do bacilo.

Em nosso estudo, observamos que houve uma intima relação entre as regiões de pobreza, representada pelos chamados "aglomerados subnormais" do município e aglomerados populacionais com um maior número de casos em três principais regiões do município.

O uso do álcool e o consumo de drogas ilícitas são considerados como fatores associados à falta de uma adesão continuada à terapêutica medicamentosa de doenças crônicas como a tuberculose, tendo se apresentado como barreira e desafio para as pessoas que vivem com HIV/AIDS e com a coinfecção tuberculose (Filho et al, 2012). Em nosso estudo, notamos que as regiões com maior número de casos de Tuberculose também eram as regiões com maior consumo de drogas, evidenciando o consumo de drogas ilícitas como uma grande barreira ao controle da Tuberculose.

\section{Considerações Finais}

O presente estudo permitiu reconhecer a situação epidemiológica espacial em que se encontra a Tuberculose por um período de dez anos no município de Santos, identificando aquelas regiões com maiores taxas de aglomeração de casos.

Foram identificadas três regiões principais que contribuíram para a maioria dos casos no município, sendo elas, a zona noroeste, a região portuária do Paquetá e a região de morros da nova Cintra e Monte Serrat. Políticas públicas voltadas para essas três regiões poderiam evitar a persistência da disseminação de casos entre elas. 
Os autores, entretanto, compreendem que analises estatísticas para correlação entre a ocorrência do evento e o espaço poderiam auxiliar na identificação, monitoramento e intervenções para reduzir o número de casos para a região.

Espera-se que este estudo traga contribuições para a população local, na medida que disponibiliza informações até então desconhecidas da distribuição espacial e das condições de vida dos doentes de $\mathrm{Tb}$, apontando áreas que devem ser consideradas prioritárias para o controle da doença. Demais trabalhos acerca deste tema poderiam explorar os casos a partir de 2016 e tentar correlacionar as variáveis que estudamos através de testes estatísticos visando encontrar associações entre as variáveis.

\section{Referências}

Arakaki-Sanchez D, Brito R. C, Brazil, organizadores. Manual de recomendações para o controle da tuberculose no Brasil. 1a edição. Brasília, DF: Ministério da Saúde, Secretaria de Vigilância em Saúde, Departamento de Vigilância Epidemiológica; 2011. 284 p. (Série A. Normas e Manuais Técnicos).

Barcellos C, \& Santos S. M (1997). Colocando dados no mapa: a escolha da unidade espacial de agregação e integração de base de dados em saúde e ambiente através do geoprocessamento. Inf Epidemiol SUS;5(1):21-9.

Barreira, D. et al (2018). Os desafios para a eliminação da tuberculose no Brasil. Epidemiologia \& Serviços de Saúde, 27(1), 1-4.

Câmara, G., Carvalho, M. S., Cruz, O. G., \& Correa, V. Análise espacial de áreas. Livro online. 2001

Chirinos, N. E. C., \& Meirelles, B. H. S (2011). Fatores associados ao abandono do tratamento da tuberculose: uma revisão integrativa. Texto contexto enferm;20(3):599-606.

Czeresnia, D et al. (1985) Considerações sobre a Tendência da Tuberculose no Brasil. Cad. Saúde Pública. 1 (3): $313-26$.

Fasca, S.F., et al (2008). Tuberculose e condições de vida: uma análise do Estado do Rio de Janeiro, Brasil, 2000 a 2002. Dissertação Doutorado em Ciências na Área de Saúde Pública. Rio de Janeiro: Escola Nacional de Saúde Pública, Fundação Oswaldo Cruz; 2008.

Filho, M. P. S., Luna, I. T, Silva, K. L. \& Pinheiro, P. N. C. (2012) Pacientes vivendo com HIV/ AIDS e coinfecção tuberculose: dificuldades associadas à adesão ou ao abandono do tratamento. Rev Gaúcha Enferm.33(2):139-145.

Hino P et al. Padrões espaciais da tuberculose associados ao indicador adaptado de condição de vida no município de Ribeirão Preto (tese). Ribeirão Preto: Escola de Enfermagem de Ribeirão Preto da Universidade de São Paulo; 2008.

Justo, M. T, Lourenção, L. G., Sasaki, N. S. G. M. dos S., Vendramini, S. H. F., De Souza, N. G., \& Santos, M. de L. S. G. (2018). Associação entre tuberculose e consumo de drogas lícitas e ilícitas. $E B .17(5): 460$

Leal, B. N. (2018) Análise espacial em tuberculose e a rede de atenção primaria em saúde. Revista Brasileira de Enfermagem.

Lapa, T., Ximenes, R. A. A., Silva, N. N., Souza, W. V., Albuquerque, M. F. M., \& Cmpozana, G. (2001) Vigilância de Hanseníase em Olinda, Brasil, utilizando técnicas de análise espacial. Cad Saúde Pública, 17:11:53-62.

Organisation mondiale de la santé, organizador. Global tuberculosis report 2017. World health organization 2017.

Rocha, C. H. B. (2000). Geoprocessamento: Tecnologia transdisciplinar. Doador;

Sales, C. M. M. Nunes, G. F. Rogério, W. Castro, T. Santos, B. R. \& Maciel, E. L. N. (2015) Tuberculose e a questão social: uma revisão sistemática de estudos brasileiros. In: Revista Brasileira de Pesquisa em Saúde. Vitória, 17 (4): 156-175

Siqueira, A. S. P. (2014) Determinantes socioeconômicos da produção da tuberculose: um estudo no município de Itaboraí, Região Metropolitana do Rio de Janeiro, no período de 2000 a 2011. Dissertação Doutorado em Ciências na Área de Saúde Pública. Rio de Janeiro: Fundação Oswaldo Cruz.

Souza, J. N., Bertolozzi M. R et al. (2007) A vulnerabilidade à tuberculose em trabalhadores de enfermagem em um hospital universitário. Ver Latino-am Enfermagem 15(2):259-266

Vicentin, G; Santo, A. H., \& Carvalho, M. S (2002). mortalidade por tuberculose e indicadores sociais no município do rio de janeiro. ciência \& saúde coletiva.

Vieira, A.G et al. (2009) Características da tuberculose pulmonar em área hiper epidêmica - município de Santos (SP). Jornal Brasileiro de Pneumologia, 35

Ximenes, R. A. A., Martelli, C. M. T., Souza, W. V. et al (1999). Vigilância de doenças endêmicas em áreas urbanas: a interface entre mapas de setores censitários e indicadores de morbidade. Cad Saúde Pública 15:53-61. 